\title{
The Role of Lifestyle Choices among Female Patients for Prevention of Coronary Artery Disease
}

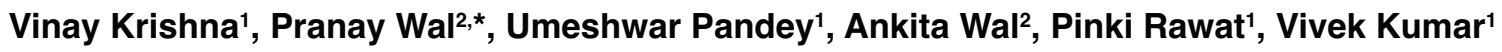 \\ ${ }^{1}$ LPS Institute of Cardiology, Ganesh Shankar Vidyarthi Memorial (GSVM) Medical College, Kanpur, Uttar Pradesh, INDIA. \\ ${ }^{2}$ Department of Pharmacy, Pranveer Singh Institute of Technology, Kanpur, Uttar Pradesh, INDIA.
}

\begin{abstract}
Objectives: There was limited data available regarding the effect of daily lifestyle choices in female patients suffering from Coronary Artery Disease (CAD). It had been found that lifestyle played a critical role in the prevention of CAD. It was evident that risk of CAD could be reduced by modifying lifestyle factors like physical activity, drinking, smoking, food, sleep patterns, etc. These were the most effective approaches to strengthen lifestyle factors. Methodology: Women from various occupational groups having CAD were selected, the total number being 109. All these patients were subjected to a routine family history of other diseases. Individual of age below 20 years were excluded from our survey. Results: The research comprised lifestyle improvement surveys which purpose was to tutor female patients regarding knowledge of the disease, their food quality, increasing physical activity, enhancing sleep patterns and leaving smoking and drinking cessations. Conclusion: It was concluded that these lifestyle choices reduce risk factors associated with CAD in female patients. A healthy diet, moderate alcohol and smoke cessations, at least 30 min of physical activity, significant sleep patterns, etc should be undertaken regularly.
\end{abstract}

Key words: Coronary artery disease, Lifestyle, Physical activity, Smoking, Drinking.

\section{INTRODUCTION}

The incidence of Coronary Artery Disease (CAD) or Ischemic Heart Disease (IHD) is increasing world while and more so in Indians. ${ }^{1,2}$ However, female CAD patients accounting for over one third of total deaths. ${ }^{3}$ Treatment includes antiplatelets, anticoagulants and other surgical methods. ${ }^{4}$ A healthy lifestyle seems to be important factor for the prevention of CAD in female patients. Various lifestyle choices like drinking, smoking, physical activity and dietary habits are healthful. ${ }^{5}$ Therefore, a healthy lifestyle could reduce relative risk of CAD. Patients were asked for their point of view regarding number of topics relating to the questions of physical activity, knowledge of accompanied disease and whether there is a role for lifestyle changes in prevention of CAD. ${ }^{6,7}$ The objective of experts was to produce best clinical practice approaches for common and complicated clinical situ- ations found in female patients associated with CAD risk. The objective of the research is to provide information and guidance for healthcare professionals on the role of lifestyle choices for the prevention of CAD in female patients. Lifestyle improvement surveys educate patients on improving knowledge of disease, diet quality, increasing physical activity and leaving smoking and drinking habits. ${ }^{8}$ These changes reduce risk factors associated with CAD (Figure 1). Therefore, the purpose of current research is to evaluate the effects of lifestyle factors on future CAD mortality.

\section{MATERIALS AND METHODS}

\section{Methodology}

\section{Study design}

Randomized control trial.
Submission Date: 12-11-2018; Revision Date: 08-01-2019; Accepted Date: 08-04-2019

DOI: 10.5530/ijper.53.3s.112 Correspondence: Dr. Pranay Wal,

Department of Pharmacy, Dean and Associate

Professor, Pranveer Singh Institute of Technology, Kanpur- 209305, Uttar Pradesh, INDIA.

Phone: +91 9795967709 drpranaywal@gmail.com 


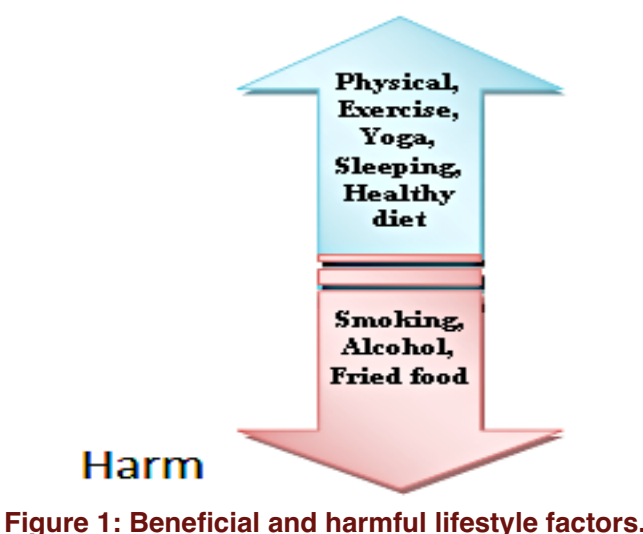

Figure 1: Beneficial and harmful lifestyle factors.

\section{Study subjects}

Women with hypertension fulfilling the inclusion criteria attending LPS Institute of Cardiology, Kanpur.

\section{Study Site}

The study was going on in the Cardiology unit of LPS Institute of Cardiology, Kanpur, under the supervision of consultant cardiologist, Dr. Vinay Krishna.

\section{Design}

Randomized pre-test, post-test controlled group study from April 2017 to April 2018.

\section{Selection of study subjects}

\section{Inclusion criteria}

Women in the age group 20 years and above.

Hypertension Stage I or II.

Giving informed written consent.

\section{Exclusion criteria}

All subjects without written consent and all subjects with secondary hypertension or known chronic disease were excluded from the study.

\section{Randomization}

Subjects were randomized into the Control and Intervention groups using block randomization technique.

\section{Intervention}

Identified initial sample of selected patients were divided into treatment group and control group by simple randomized technique. Patients in the treatment group were provided with written health education material which was modified and validated by physicians.

The base line blood pressure readings were taken for both the treatment group and control group. Then the counseling and care was provided to the treatment group. Participant goal for the intervention group would be as follows: at least $180 \mathrm{~min} / \mathrm{wk}$ of moderate-inten- sity physical activity. Participant goals to accomplish the Dietary Approach to Stop Hypertension (DASH) diet would be as follows: Increased consumption of fruits and vegetables (9-12 servings/d) and low-fat dairy products (2-3 servings/d).

\section{Follow up}

The Participants state of improvement was followed up at the OPD (Out Patient Department) visit or time of readmission if any during the study period, or through visiting their houses and contacting them through mobile phones. Then the readings at the follow ups were compared with the base line reading. The drug related problems would also be identified during the follow ups and would be resolved by discussion with the physicians.

Women from various occupational groups having CAD were selected, the total number being 109. Individuals who were ready to answer the questionnaire were enrolled in our study. All these patients were subjected to a routine family history of other diseases. Individual of age below 20 years were excluded from the survey. A questionnaire leaflet of Knowledge, Attitude and Practice (KAP) of 23 questions was framed. The questions were based on basic life style (exercise, smoking, etc), health of patients, psychology of patients for answering any given question and to get idea what the basic information a patient have about hypertension and its related factors.

\section{RESULTS AND DISCUSSION}

The responses of the patients to the questions were evaluated for knowledge, attitude and practice separately.

\section{Knowledge aspect}

There were a total of 10 questions addressing the knowledge aspect related to the disease. The patient's who had given right answers to these questions is represented below (Figure 2). It was observed that the right answers were more in the intervention group patients at the time of follow up in comparison to that in control group (Figure 3). Total percentage of patients who had given right answers to the questions of knowledge aspect is represented in the Table 1.

Physical activity related questions helped in evaluating the patient's attitude towards the disease. The patient's responses to these questions were represented in Figure 4 and 5 . It had been shown that number of right answers were more in the intervention group patients at the time of follow up. Total percentage of patients who had given right answers to the questions of Attitude aspect is represented in the Table 2. 


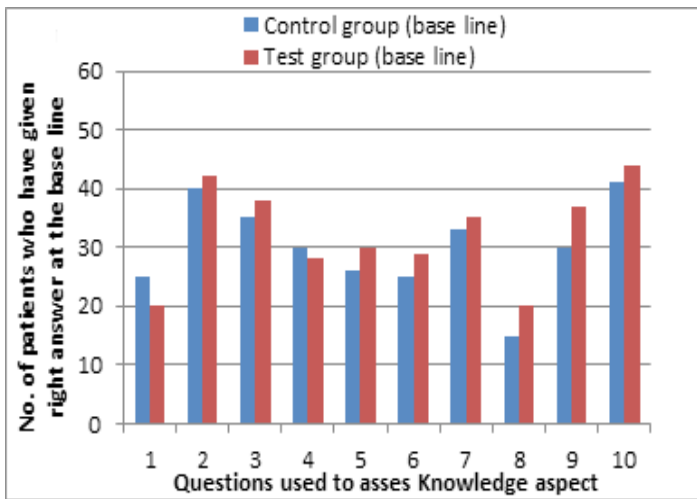

Figure 2: Number of patients who had given right answer at base line itself.

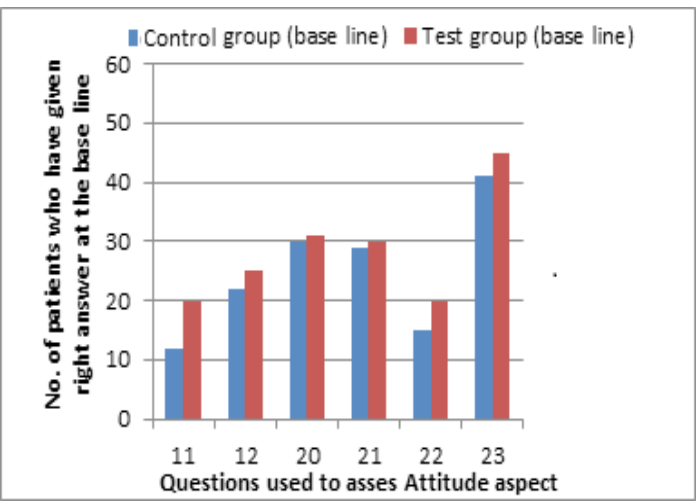

Figure 4: Number of patients who have given right answer at base line itself.

\begin{tabular}{|c|c|c|c|c|}
\hline \multicolumn{2}{|c|}{ Table 1: Percentage evaluation of knowledge aspect } \\
of kap questionnaire.
\end{tabular}

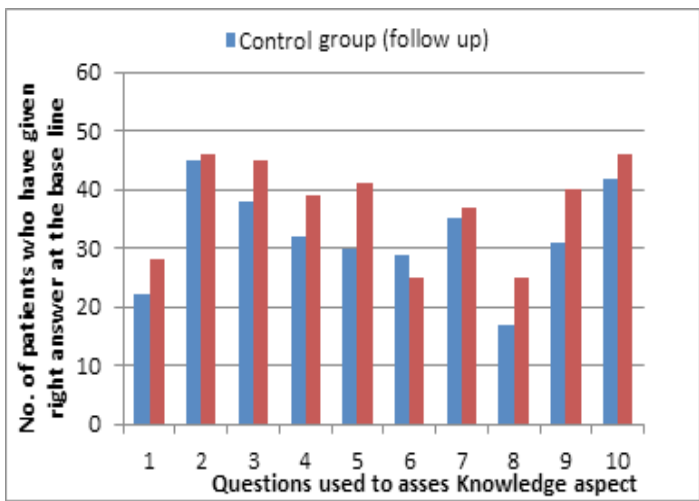

Figure 3: Number of patients who have given right answer at the follow-up.

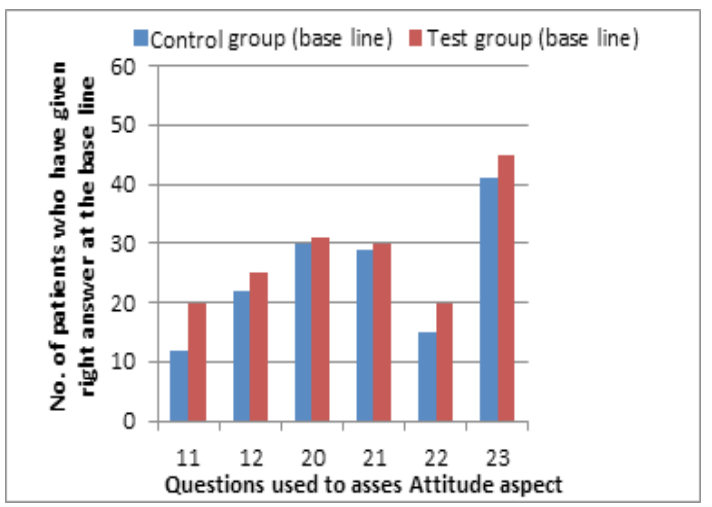

Figure 5: Number of patients who have given right answer at the follow-up.

\begin{tabular}{|c|c|c|c|c|}
\hline \multirow[t]{2}{*}{ Questions } & \multicolumn{2}{|c|}{ Baseline (\%) } & \multicolumn{2}{|c|}{ Follow up (\%) } \\
\hline & Test & Control & Test & Control \\
\hline Question no.11 & $51 \%$ & $65 \%$ & $90 \%$ & $78 \%$ \\
\hline Question no.12 & $62 \%$ & $70 \%$ & $82 \%$ & $80 \%$ \\
\hline Question no.20 & $81 \%$ & $91 \%$ & $100 \%$ & $96 \%$ \\
\hline Question no.21 & $76 \%$ & $85 \%$ & $93 \%$ & $95 \%$ \\
\hline Question no.22 & $40 \%$ & $44 \%$ & $76 \%$ & $83 \%$ \\
\hline Question no.23 & $100 \%$ & $98 \%$ & $100 \%$ & $100 \%$ \\
\hline
\end{tabular}

\section{Practice aspect}

These questions helped in evaluating the patients general Practice in day to day life. It had displayed that more patients in the intervention group quit smoking and alcohol at the time of follow up. Figure 6 and 7 compared the effect of intervention on the Practice aspect of the patients in both intervention and control group. It was observed that in the intervention group there was improvement in all the aspect of practice towards the disease. Table 3 depicted an interpretation of lifestyle aspects for prevention of CAD in female patients. 


\begin{tabular}{|c|c|c|}
\hline \multirow{2}{*}{ Lifestyle choices } & Risk & Interpretation of lifestyle aspects \\
& & Threat \\
\cline { 2 - 3 } & Occasionally & $>2 \mathrm{~h} / \mathrm{wk}$ of moderate activity \\
\hline Physical activity & Ever smoked & High intake $(<1$ drink/wk $)$ \\
\hline Alcohol Intake & Occasionally fried foods & Regular smoked $(<1 \mathrm{smoke} / \mathrm{wk})$ \\
\hline Smoking & 3 to $5 \mathrm{hr}$ & $>3 \mathrm{hr}$ \\
\hline Balanced diet & & Weekly fried foods \\
\hline Sleep &
\end{tabular}

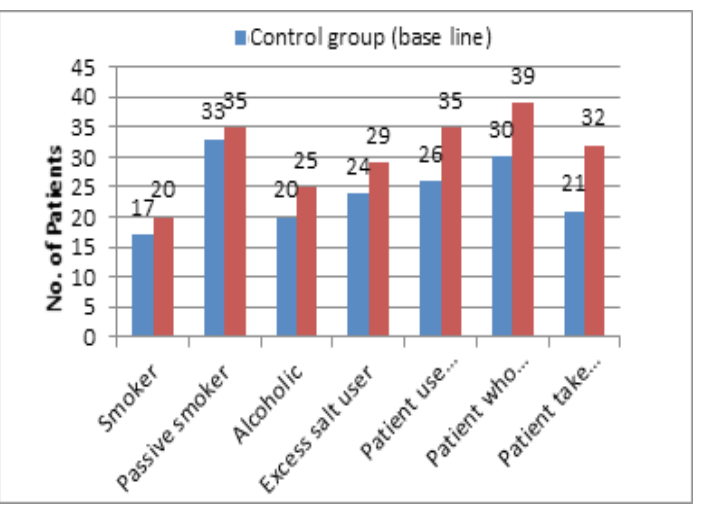

Figure 6: Number of patients who have given right answer at base line itself.

\section{CONCLUSION}

It was concluded that lifestyle improvements could lead to significant CAD reductions in female patients. A healthy diet should be recommended and should be adapted by every patient. It is suggested that moderate alcohol consumption, could have a negative impact on patient's health, although everyday consumption was a threat to patient. At least 30 min of physical activity should be undertaken daily. It is recommended that patients should leave or suppress drinking and smoking intake. Sleep deprivation could also result in adverse CAD mortality.

\section{ACKNOWLEDGEMENT}

This work is acknowledged to the Indian Council of Medical Research (ICMR), New Delhi, India, for their financial support.

\section{CONFLICT OF INTEREST}

The authors declare no conflict of interest.

\section{ABBREVIATIONS}

CAD: Coronary artery disease; IHD: Ischemic heart disease; LPS: Institute of Cardiology; DASH: Dietary

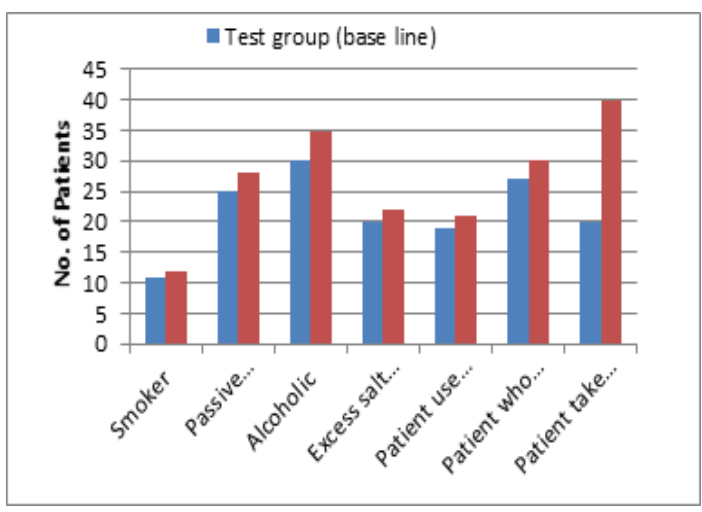

Figure 7: Number of patients who have given right answer at the follow-up.

approach to stop hypertension; OPD: Outpatient department; KAP: Knowledge, attitude and practice.

\section{REFERENCES}

1. Wan YF, Ma XL, Yuan C, Fei L, Yang J, Zhang J. Impact of daily lifestyle on coronary heart disease. Exp Ther Med. 2015;10(3):1115-20.

2. Omole MK, Bello DE. Case Studies Assessment on Rational Use of Drugs among Patients with Ischemic Heart Disease at a Tertiary Hospital in South West Nigeria. Asian J Pharm Clin Res. 2012;5:20-5.

3. Pathak LA, Shirodkar S, Ruparelia R, Rajebahadur J. Coronary artery disease in women. Indian Heart J. 2017;69(4):532-8.

4. Thamayanthi K, Ranganathan A, Vasanthira K. Effect of Tirofiban on the outcome during Hospitalization in Patients with Acute Coronary Syndrome an Observational Study. Asian J Pharm Clin Res. 2018;11(8):388-91.

5. Puddu PE, Menotti A. The impact of basic lifestyle behaviour on health: How to lower the risk of coronary heart disease, other cardiovascular diseases, cancer and all-cause mortality. Lifestyle adaptation: A global approach. E-Journal of Cardiology Practice. 2015;13(32):29.

6. Masana L, Ros E, Sudano I, Angoulvant D. The lifestyle expert working group. Is there a role for lifestyle changes in cardiovascular prevention? What, when and how?. Atherosclerosis Supp. 2017;26:2-15.

7. Odegaard AO, Koh WP, Gross MD, Yuan JM, Pereira MA. Combined Lifestyle Factors and Cardiovascular Disease Mortality in Chinese Men and Women the Singapore Chinese Health Study. Circulation. 2011;124(25):2847-54.

8. Staimez LR, Weber MB, Gregg EW. The Role of Lifestyle Change for Prevention of Cardiovascular Disease in Diabetes. Curr Atheroscler Rep. 2014;16(12):460. 


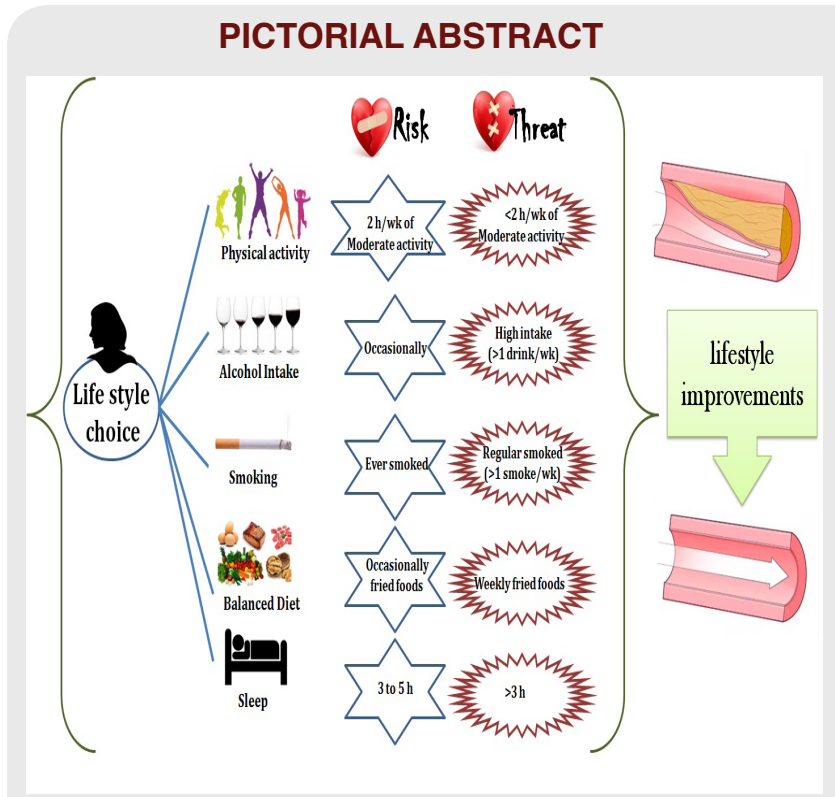

About Authors

\section{SUMMARY}

Women who were having CAD and ready to answer the questionnaire were enrolled in current survey study. All these patients were subjected to a routine family history of other diseases. Individual of age below 20 years were excluded from the survey. A questionnaire leaflet having 23 questions of Knowledge, Attitude and Practice (KAP) was framed. The questions were based on basic life style (exercise, smoking, etc), health of patients, psychology of patients for answering any given question and to get idea what the basic information a patient had about the hypertension and its related factors. It had been found that these lifestyle choices significantly reduce risk factors associated with CAD in female patients. A patient should have to follow a healthy diet style pattern, moderate alcohol and smoke cessations, at least 30 min of physical activity, significant sleep patterns, etc.

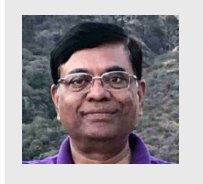

Dr. Vinay Krishna: One of the top Pulmonologists Doctors, Kanpur. He has completed MBBS, MS (Gen Surg), MCH (CTVS), Cardiovascular and Thoracic Surgery from King George Medical University, Lucknow during 1975-1986. Currently he is working as Director in LPS Institute of Cardiology and Cardiac Surgery, Kanpur since last 30 years. His skills and expertise are on Heart Failure, Atrial Fibrillation, Cardiac Surgery, Pacemakers, Airway Obstruction, Aortic Diseases, Vascular Surgery, Heart Valve Diseases, Cardiovascular Surgery.

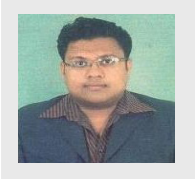

Dr Pranay Wal, working as an Associate Professor and Dean research and development in PSIT Kanpur. He has working on various Indian government funded projects (ICMR, UPCST, DST etc). He has published more than 25 research articles and chapter in books published by Elsevier. Member of professional bodies like International pharmaceutical federation, ISPOR, APTI etc. He have filled 3 patents, and involved in designing new technologies for health care.

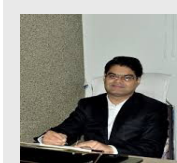

Dr. Ummeshwar Pandey, working as an Associate Professors at, L.P.S Institute of Cardiology, Kanpur. He has completed his studies at Ganesh Shankar Vidyarthi Memorial Medical College. He has working on government projects. He has great knowledge in Hypertension, Myocardial Infarction Insulin, Coronary Artery Disease. He has published more than 10 articles.

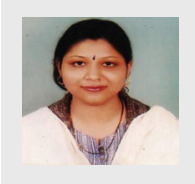

Dr. Ankita Wal has expertise in Biostatistics. She had completed her Ph.D in Pharmaceutical Chemistry. She is working on Indian Council of Medical Research project on effect of stress and diet on development of cardiac disease. She has published more than 20 national and international papers in reputed journals. She has filed two patents till date. She is serving as a Reviewer form International Journal of Pharmaceutical Science and Biotechnology, Journal of Pharmaceutical Science and Research, Serial Publications etc. She is constantly writing chapters in books for Elsevier and has published four books till date.

Cite this article: Krishna V, Wal P, Pandey U, Wal A, Rawat P, Kumar V. The Role of Lifestyle Choices among Female Patients for Prevention of Coronary Artery Disease. Indian J of Pharmaceutical Education and Research. 2019;53(3 Suppl 2):s399-s403. 